uncommon: cirrhosis develops in fewer than $3 \%$ of patients with inflammatory bowel disease. ${ }^{111} 12$ Chronic active hepatitis, amyloidosis, granulomas, and hepatic abscesses are also rare, and taken together occurred in only $1-2 \%$ of patients in several large series. ${ }^{1}$ 9-12

These reassuring figures have been confirmed in a recent survey from Birmingham of over 1200 patients with inflammatory bowel disease. ${ }^{13}$ Abnormal liver function tests were present at some time in just over $8 \%$ of patients, but in many cases they developed transiently after surgery and were associated with abdominal sepsis. One-fifth of patients with abnormal liver function tests had pericholangitis. Cirrhosis was found in three of the 517 patients with Crohn's disease and in 11 of the 720 cases of ulcerative colitis, giving an overall prevalence of about $1 \%$.

The cause of chronic liver disease in these patients remains obscure. It is not related to blood transfusion, drug treatment, or other systemic complications. ${ }^{9}$ Portal bacteraemia has been suggested as a possible mechanism, but cultures of liver biopsy specimens and portal blood samples are usually sterile. ${ }^{40-12}$ Moreover, pericholangitis progresses to cirrhosis only rarely. The effect of colectomy is uncertain, for, although liver histology may improve after resection, ${ }^{14}$ differences in biopsy technique, sampling error, and possible observer bias make this observation difficult to interpret-and we have to remember that patients with cirrhosis may enjoy good health for many years after colectomy. Although resection may retard or reverse the progression of the liver disease (or delay the onset of hepatic encephalopathy), possibly also cirrhosis associated with inflammatory bowel disease runs a particularly slow course or laparotomy leads to the detection of liver disease at a very early stage.

Gall stones are present in up to a third of patients with Crohn's disease of the terminal ileum-at least three times the prevalence in control subjects. ${ }^{4}$ Ileal disease or resection results in the malabsorption of bile salts with reduction in their pool and supersaturation of the bile with cholesterol. ${ }^{15}$ The tenfold increase in these patients in the incidence of carcinoma of the bile ducts and gall bladder is more difficult to explain. The Birmingham workers found such neoplasms in eight of their patients with ulcerative colitis. They differ from the usual carcinomas of the biliary tract by developing in younger patients without gall stones, and one puzzling feature is that they may develop many years after colectomy.? Sclerosing cholangitis may affect the intrahepatic or extrahepatic biliary tree, with diffuse narrowing of the bile ducts or multiple strictures separated by saccular dilatation, ${ }^{6}$ and is sometimes associated with finding pericholangitis in the liver biopsy sample. ${ }^{16}$ Sclerosing cholangitis was diagnosed in seven $(1 \%)$ of patients with ulcerative colitis in the Birmingham series.

How can we recognise these disorders? Although liver function tests may be normal in patients with fatty liver, pericholangitis, or even cirrhosis, measurement of serum enzyme concentrations such as alkaline phosphatase remains the best screening test generally available. Serious liver disease is more common in patients with extensive disease of the colon and a reasonable plan would be to check liver function tests every 12 months in such patients. A rise in serum enzyme concentrations or the development of pain in the right upper quadrant of the abdomen, enlargement of the liver, jaundice, or pruritus would all indicate the need for further investigation; and if the patient has had a colectomy (no matter how many years previously) the possibility of a bile duct carcinoma should be considered. Although liver biopsy is safe in patients with obstruction of the large ducts, ${ }^{17}$ radiographic assessment of the biliary tree will be more appropriate in most cases. Intravenous cholangiography may be unsuccessful in patients with jaundice, when ultrasonography may be a helpful non-invasive test to determine whether or not the bile ducts are dilated. Percutaneous transhepatic cholangiography will outline the biliary tree in over $90 \%$ of patients with dilated ducts, but endoscopic retrograde cholangiography is the more useful procedure in those with non-dilated ducts. ${ }^{18}$

The radiographs may show gall stones or a single neoplastic stricture, either of which may be treated surgically. The management of sclerosing cholangitis is less satisfactory. Steroids, azathioprine, and antibiotics have all been used but are of uncertain benefit. ${ }^{619}$ Dilatation of the extrahepatic bile ducts with insertion of a $\mathrm{T}$-tube for several months may allow inflammation to resolve without the formation of new strictures. ${ }^{20}$ Nevertheless, the outlook for most patients with sclerosing cholangitis is poor: they may develop ascending bacterial infection, progressive jaundice, intractable pruritus, and secondary biliary cirrhosis.

\footnotetext{
${ }^{1}$ Greenstein, A J, Janowitz, H D, and Sachar, D B, Medicine (Baltimore), 1976, 55, 401 .

${ }^{2}$ Mistilis, S P, Annals of Internal Medicine, 1965, 63, 1.

3 Watts, H D, American fournal of Digestive Diseases, 1978, 23, $41 \mathrm{~s}$

4 Cohen, S, et al, Gastroenterology, 1971, 60, 237.

5 Baker, A L, et al, American fournal of Digestive Diseases, 1974, 19, 109.

${ }^{6}$ British Medical fournal, 1976, 2, 1090.

${ }^{7}$ Ritchie, J K, et al, Quarterly fournal of Medicine, 1974, 43, 263.

8 Akwari, O E, et al, Annals of Surgery, 1975, 181, 303.

9 Eade, M N, Annals of Internal Medicine, 1970, 72, 475

${ }_{10}$ Eade, M N, et al, Annals of Internal Medicine, 1971, 74, 518.

11 Perrett, A D, et al, Quarterly fournal of Medicine, 1971, 40, 211.

12 Perrett, A D, et al, Quarterly fournal of Medicine, 1971, 40, 187.

${ }^{13}$ Dew, M J, Thompson, H, and Allan, R N, Quarterly fournal of Medicine, $1979,48,113$

${ }^{14}$ Eade, M N, Cooke, W T, and Brooke, B N, Annals of Internal Medicine, 1970, 72, 489

${ }^{15}$ Marks, J W, et al, American fournal of Digestive Diseases, 1977, 22, 1097

${ }^{16}$ Blackstone, M O, and Nemchausky, B A, American fournal of Digestive Diseases, 1978, 23, 579.

17 Morris, J S, et al, Gastroenterology, 1975, 68, 750

18 Elias, E, et al, Gastroenterology, 1976, 71, 439.

19 Mistilis, S P, Skyring, A P, and Goulston, S J M, Australasian Annals of Medicine, 1965, 14, 286.

20 Schwartz, S I, The Surgical Clinics of North America, 1973, 53, 1161.
}

\section{Wanted: a new wound dressing}

With the notable exception of the burn, for which the late A B Wallace successfully reintroduced open treatment in the late 1940 s, our natural inclination is to cover any wound. Paré's over-quoted statement "I dressed him; God healed him" attests to the orthodoxy of a practice that goes back at least to the Smith papyrus. Though in each generation some surgeons expose the sutured wound to the air, with or without some ritualistic practice such as a spray of fast-drying plastic, they are the exception. Perhaps dressings are more social than surgical, on the one hand preserving clothes and bed linen from blood and exudate and on the other hand shielding the patient from the affront to his body's integrity.

If wounds are to be dressed then let it be with the most efficient materials. T D Turner ${ }^{1}$ at the Surgical Dressing Research Unit of the Welsh School of Pharmacy has recently looked at the types of dressing required and the commonly available products ostensibly designed to meet the clinical need. He distinguishes arbitrarily but conveniently between dressings 
predominantly used for closed wounds and those used for open wounds. The latter may be combined with wet applications (for example, Eusol or hydrogen peroxide) or have a "dry" pre-impregnation (for example, soft paraffin). A survey of use showed a wide variety of both kinds throughout an unspecified number of hospitals in Europe, with not unexpectedly gauze predominating for closed wounds and dry dressings for exudative wounds. Turner next went on to match the products in common use to need, particularly absorption. In his view, gauze performs poorly and is abrasive to the open wound, as are the many composite materials, which all stem from the remarkable invention of Joseph Gamgee. The more complicated modern dressing incorporates nonadherent film, which, though it leads to greater comfort for the patient, has not solved the problems of inadequate absorptive capacity and therefore "strike-through," loss of the nonadherent surface, fibre shedding into the wound, inadequate moulding to the body surface, and illogical sizes.

What we need is a new dressing that has a stable, nonadherent contact layer which allows a humidity gradient as well as gas exchange and a better absorptive mass superficially. A flexible pad of viscose fibres with a heat-bonded polyethylene surface seems to be the best approach and is under development. If dressing technology could be rationalised and simplified by such a material much time and money could be saved in our hard-pressed Health Service.

1 Turner, T D, Pharmaceutical fournal, 1979, 222, 421.

\section{Bronchopulmonary lavage}

Bronchopulmonary lavage has been used for more than ten years in specialist centres treating chronic lung diseases, and interest in the technique is now growing rapidly. In pulmonary alveolar proteinosis, for example, the technique of bronchial lavage ${ }^{1}$ is to isolate the two lungs by a double-lumen tracheobronchial catheter, and to anaesthetise, ventilate, and oxygenate the patient through one channel while using the other to wash out the opposite lung with buffered normal saline at body temperature. After ventilation with $100 \%$ oxygen for 10 minutes has "denitrogenised" the lung its complete functional residual capacity may be replaced by the saline as the oxygen is absorbed. Thereafter, amounts of saline equivalent to the tidal volume are repeatedly exchanged by gravity up to a total of 20 litres. Large quantities of lipid-rich proteinaceous material and cellular debris are removed with the effluent. The procedure may then be repeated a few days later on the other lung. Though more than one treatment may be required, dramatically successful results have been obtained in some cases, ${ }^{2}$ and prolonged survival, or even complete recovery, is now possible from a potentially fatal disease.

The same technique would be considerably more hazardous in patients with very severe acute asthma, who may become critically hypoxic if one lung is rendered functionless. In such patients, however, a different method of bronchial lavage ${ }^{3}$ may be life saving when extensive bronchial obstruction by mucous plugs or casts is preventing a patient from being adequately ventilated and oxygenated despite intubation. Volumes of $30 \mathrm{ml}$ of saline (to a total of $500 \mathrm{ml}$ ) are squirted down each main bronchus under intravenous anaesthesia and then removed by suction through an aspirating tube. Numerous casts can often be removed with subsequent relief of airways obstruction. Good results have also been reported when this technique has been used in patients-with intractable chronic asthma resistant to corticosteroids. ${ }^{4}$

Such good results from pulmonary and bronchial lavage in asthmatics and others have stimulated interest in its research potential. Cytological, chemical, and histochemical investigations may all yield valuable data from bronchoalveolar washings. Diagnostic lavage carried out by fibreoptic bronchoscopy is relatively non-invasive and can provide information about living cells, particularly the scavenging and secretory activities of macrophages ${ }^{5}$; alveolar macrophage-lymphocyte interaction ${ }^{7}$; and the effects on these processes of drugs such as halothane ${ }^{9}$ and lignocaine, ${ }^{10}$ and of noxious agents such as bacterial endotoxins ${ }^{11}$ and tobacco smoke. ${ }^{12}$ Since serial samples can be obtained bronchoalveolar lavage can be used not only to assess the state of cellular and humoral lung defences against inhaled allergens, bacteria, inorganic particles, and drugs, but also, for example, to study the evolution of the pathological changes resulting from cigarette smoking. Examination of bronchoalveolar washings may eventually serve as a useful adjunct, or even as an alternative, to lung biopsy in the diagnosis and management of certain types of diffuse pulmonary disease, such as pneumoconiosis, sarcoidosis, ${ }^{13}$ and fibrosing alveolitis. ${ }^{13} 14$

Enthusiasm for the diagnostic and research potential of bronchoalveolar lavage is now running high in academic respiratory centres, and many of the references ${ }^{5-14}$ cited in this article are to preliminary communications given at the 21st Aspen Lung Conference in 1978 and the Meeting of the Thoracic Society in February 1979. The information is still fragmentary and often confusing; but this new approach to the investigation of normal and abnormal alveolar cellular function may soon lead to major advances in our understanding of the lung diseases.

\footnotetext{
1 Ramirez-R, J, Diseases of the Chest, 1966, 50, 581.

2 Costello, J F, et al, Thorax, 1975, 30, 121.

${ }^{3}$ Marchand, P, and Van Hasselt, H, Lancet, 1966, 1, 227.

4 Thompson, H T, and Pryor, W J, Lancet, 1964, 2, 8.

${ }^{5}$ Davis, G S, Brody, A R, and Adler, K B, Chest, 1979, 75 (suppl), 280

${ }^{6}$ Du Bois, R, Thorax, 1979, 34, 424.

7 Yeager, H, jun, Lussier, L and Prashad, J, Chest, 1979, 75 (suppl), 289.

${ }^{8}$ Lawrence, E C, et al, Chest, 1979, 75 (suppl), 279.

9 Manawadu, B R, and LaForce, F M, Chest, 1979, 75 (suppl), 242.

${ }^{10}$ Hoidal, J R, White, J G, and Repine, J E, Chest, 1979, 75 (suppl), 243.

${ }^{11}$ Rylander, R, Mattsby, I, and Snella, M-C, Chest, 1979, 75 (suppl), 278.

12 Haslam, P L, Thorax, 1979, 34, 423.

13 Hunninghake, G, et al, Chest, 1979, 75 (suppl), 266

14 Turton, C W G, et al, Thorax, 1979, 34, 424.
}

\section{A critical time for diagnostic imaging}

No doctor can reasonably expect to spend a lifetime in clinical work without adjusting - however reluctantly-to changes in medical practice. Diagnostic imaging is more susceptible to technical changes than are most specialties. The present rate of change, with demand increasing faster than capacity and with new imaging techniques maturing, is creating serious problems.

Earlier this year a group of "critical radiologists," with representatives of the Royal College of Radiologists, met in Cambridge to discuss problems and solutions. The approaching crisis essentially stems from the discrepancy between 